\title{
Hypochlorous Acid Staining with R19-S in the Drosophila Intestine upon Ingestion of Opportunistic Bacteria \\ Salma Hachfi ${ }^{1,2}$, Olivia Benguettat ${ }^{1,2}$ and Armel Gallet ${ }^{1,2, *}$
}

\author{
${ }^{1}$ Université Côte d'Azur, CNRS, INRA, ISA, France; 'Institut Sophia Agrobiotech, UMR CNRS \\ 7254/INRA 1355/UNS, 400 route des Chappes, BP 167, 06903 Sophia Antipolis Cedex, France \\ *For correspondence: gallet@unice.fr
}

\begin{abstract}
[Abstract] The intestine is endowed with an innate immune system that is required to fight any exogenous bacteria that are swallowed along with the food. The first line of defense that is mounted by the gut epithelium is the release of immune Reactive Oxygen Species (ROS), such as hypochlorous acid $(\mathrm{HOCl})$, into the lumen. $\mathrm{HOCl}$ is produced within $1.5 \mathrm{~h}$ of bacterial ingestion and is very labile once released. Therefore, to monitor $\mathrm{HOCl}$ production upon ingestion of allochthonous bacteria, one needs a detection system that can quickly and efficiently detect $\mathrm{HOCl}$ production in the intestine. While most of the ROS-sensitive probes available in the market detect all kinds of ROS without any distinction, the R19-S fluorescent probe has been developed to specifically detect $\mathrm{HOCl}$. Here, we describe a protocol to monitor $\mathrm{HOCl}$ production using this probe in the gut lumen of adult Drosophila upon ingestion of the opportunistic bacteria Bacillus thuringiensis.
\end{abstract}

Keywords: Intestine, Innate immune system, Reactive Oxygen Species, $\mathrm{HOCl}$, Opportunistic bacteria, Drosophila melanogaster

[Background] The evolutionarily conserved innate immune system is the first line of defense against pathogenic bacteria. In the gut, the local innate immune system first produces Reactive Oxygen Species (ROS) to damage bacterial wall and slow down the proliferation of ingested bacteria (Kim and Lee, 2014). Then, the visceral mesoderm triggers spasmodic contractions to favor bacterial eviction (Benguettat et al., 2018). The third line of defense is the production of antimicrobial peptides that kill the bacteria (Xia et al., 2017). Finally, the gut is able to regenerate damaged epithelial cells to maintain gut integrity, avoiding bacterial entry into the internal milieu (Bonfini et al., 2016). In Drosophila, the immune ROS are produced by the enterocytes of the anterior midgut in a DUOX-dependent manner between 0.5 and 1.5 $\mathrm{h}$ after bacterial ingestion. The main immune ROS to be released in the gut lumen is hypochlorous acid $(\mathrm{HOCl})$ (Lee et al., 2013; Benguettat et al., 2018). Therefore, $\mathrm{HOCl}$ monitoring is a good proxy for the gut innate immune response to a microbe. However, $\mathrm{HOCl}$ is a very labile compound that quickly disappears once it is released into the lumen. Here, we describe a protocol that we designed to efficiently detect $\mathrm{HOCl}$ production in the gut lumen of adult Drosophila upon ingestion of low doses of the Grampositive Bacillus thuringiensis kurstaki (Btk) opportunistic bacteria. This protocol can equally be used to detect $\mathrm{HOCl}$ upon ingestion of any other pathogens and it also can be used to measure $\mathrm{HOCl}$ production in the gut of Drosophila larvae. 


\section{Materials and Reagents}

\section{A. Drosophila rearing}

1. 6 oz Drosophila stock bottles (Genesee Scientific, catalog number: 32-130)

2. Cotton balls for stock bottles (Genesee Scientific, catalog number: 51-102B)

3. Cantons flies (Bloomington Drosophila Stock Center, catalog number: 64349) (flystocks.bio.indiana.edu)

4. Agar (VWR, BDH ${ }^{\circledR}$, catalog number: 20768-361)

5. Sugar (Carrefour or any other supermarket)

6. Cornflour (AB, Celnat-NaturDis)

7. Yeast (Biospringer, catalog number: BA10/0-PW)

8. Tegosept (Apex, Fly Food preservative, Genesee Scientific, catalog number: 20-258)

9. Standard nutrient medium for Drosophila (see Recipes)

B. Bacterial culture

1. Petri dishes (Thermo Scientific-Nunc ${ }^{\top M}$, catalog number: 263991)

2. Sterile tips (Sigma Aldrich, catalog number: CLS4138)

3. $15 \mathrm{ml}$ tubes (Corning, Falcon ${ }^{\circledR}$, catalog number: 352096)

4. Graduated cylinder (Azlon, catalog number: 11940985)

5. Bacillus thuringiensis var. kurstaki (Btk) strain identified under the code 4D22 at the Bacillus Genetic Stock Center (http://www.bgsc.org/) and described by Gonzalez et al., 1982

6. Luria broth powder (Conda, catalog number: 1551)

7. Agar bacteriological (Euromedex, catalog number: 1330)

8. LB medium (see Recipes)

9. LB-agar medium (see Recipes)

C. Inoculation

1. Cotton balls for $25 \mathrm{~mm}$ narrow vials (Genesee Scientific, catalog number: 51-101)

2. Spectrophotometry cuvettes (Ratiolab, catalog number: 2712120)

3. $2 \mathrm{ml}$ microtubes (Paul Boettger, catalog number: 02-043)

4. $20 \mathrm{~mm}$ filter disks (3 MM Chr chromatography paper; GE Healthcare, catalog number: 3030917)

5. $50 \mathrm{ml}$ tubes (Fisher Scientific, catalog number: 10788561

6. $25 \mathrm{~mm}$ narrow Drosophila vials (Genesee Scientific, catalog number: 32-109RL)

7. Sucrose (Euromedex, catalog number: 200-301-B)

8. $5 \%$ sucrose (see Recipes)

9. 1 mM R19-S (Futurechem, FC-8001, Seoul, South Korea) (see Recipes) 
D. Dissection

1. 10x PBS (Euromedex, catalog number: ET330)

2. $1 x$ phosphate-buffered saline (PBS) (see Recipes)

E. Assay

1. $1.5 \mathrm{ml}$ microtubes (Paul Bottger, catalog number: 02-063)

2. 12-well black microplates (VWR international, catalog number: 734-2324)

3. Aluminum foil (available at any supermarket)

4. Formaldehyde $16 \%$ (Thermo Scientific, catalog number: 28908)

5. Vectashield/DAPI (Sigma, catalog number: F6057)

6. $4 \%$ Formaldehyde (see Recipes)

\section{Equipment}

A. Drosophila rearing

Refrigerated oven at a constant temperature of $25^{\circ} \mathrm{C}$ and with a $12 \mathrm{~h} / 12 \mathrm{~h}$ light/dark cycle (Fisher Scientific, catalog number: 11857552). Humidity must be maintained between $40 \%$ and $70 \%$.

B. Bacterial culture

1. $500 \mathrm{ml}$ sterile flask

2. $30^{\circ} \mathrm{C}$ shaking incubator (Infors, model: AK 82)

C. Inoculation

1. Spectrophotometer (Aqualabo, Secomam, model: Prim Light \& Advanced)

2. $\mathrm{CO}_{2}$-anesthesia system (INJECT+MATIC sleeper)

D. Dissection

1. Dumont forceps \#5 (Fine Science Tools, catalog numbers: $11251-20$ and 11252-20)

2. Watch glass (Steriplan Petri dishes, DWK Life Sciences, catalog number: 237554008)

3. Stereomicroscope (Leica Microsystems, model: Leica M60)

E. Assay

Florescent microscope (Zeiss Axio Imager Z1 Apotome) equipped with a camera (AxioCam MRm) and a Rhodamine 43 fluorescent filter

\section{Software}

1. ZEN 2012 (Zeiss)

2. ImageJ (https://imagej.nih.gov/ij/) 
3. Kyplot (http://kyenslab.com/en/index.html)

4. Excel (Microsoft)

\section{Procedure}

A. Drosophila rearing

1. Canton $\mathrm{S}$ flies (Bloomington) are reared on standard medium for Drosophila melanogaster (see Recipes) at $25^{\circ} \mathrm{C}$.

2. To obtain synchronized mated females, remove adult flies from rearing bottles and wait for the emergence of new flies for one day. Then, transfer the newly emerged flies (males and females) to new bottles for five more days at $25^{\circ} \mathrm{C}$ before inoculation. In our experiments, five to six-dayold mated females were used.

Note: If you want to work only on virgin females, once new flies have hatched, remove the males immediately and place the virgin females in a separated vial until they reach the correct age.

B. Bacterial culture

In the experiments presented below, flies were inoculated with the Btk bacterial strain (see Materials and Reagents). A solution of $5 \%$ sucrose serves as the negative control (Ctrl).

1. Spread bacteria from the stock onto LB agar Petri dishes and grow overnight at $30^{\circ} \mathrm{C}$.

2. Pick a single colony using a sterile tip and inoculate $250 \mathrm{ml}$ of LB in a $500 \mathrm{ml}$ sterile flask, and incubate overnight at $30^{\circ} \mathrm{C}$ with shaking at $180 \mathrm{rpm}$.

Note: The growth of Btk is very efficient. There is no need for a starter culture.

C. Inoculation

1. On the day of infection, separate 5 to 6 -day-old females from males and put into a vial without medium for a $2 \mathrm{~h}$ starvation period at $25{ }^{\circ} \mathrm{C}$. This ensures the synchronization of food intake when they are introduced to Medium with bacteria.

2. During this time, measure the optic density (OD) of the overnight bacterial culture. If necessary, dilute the overnight culture to get an OD between 0.2 and 0.8 .

3. Preparation of the bacterial solution: the required $\mathrm{OD}$ to inoculate flies with $10^{8}$ Colony Forming Unit (CFU) of Btk per Drosophila is presented in Table 1.

Table 1. Bacterial solution

\begin{tabular}{|l|l|l|l|l|}
\hline Bacterium & $\begin{array}{l}\text { Volume to place } \\
\text { on the media }\end{array}$ & Required OD & $\begin{array}{l}\text { CFU/vial } \\
\text { (for } 10 \text { flies/vial) }\end{array}$ & CFU/fly \\
\hline Btk & $25 \mu \mathrm{l}$ & 400 & $1 \times 10^{9}$ & $1 \times 10^{8}$ \\
\hline
\end{tabular}

Notes: 
a. The OD of the overnight culture will be obviously too low and you must concentrate it. Pellet the bacterial culture by spinning at 2,500 $\mathrm{g}$ for $15 \mathrm{~min}$ at room temperature. Resuspend the pellet with the required volume of $5 \%$ sucrose to get the correct CFU according to the formula $f O D \times f V=i O D \times i V(f O D=$ final $O D ; i O D=$ initial $O D, f V=$ final Volume; iV= initial Volume). For example, to obtain an $O D 400$ (fOD) of Btk bacteria from an overnight culture at $O D 2$ ( $(O D)$, spin $25 \mathrm{ml}$ of the bacterial culture (iV) and resuspend the pellet in a volume of $5 \%$ sucrose that is $200 x$ less than the initial volume of $L B$, and thus $f V=125 \mu$.

b. One can inoculate flies with any other bacteria. However, depending on the type of bacteria, the $O D$ that is required to yield the desired CFU will be probably different.

4. Dilute the $1 \mathrm{mM}$ stock solution of R19-S (see Recipes) to $100 \mu \mathrm{M}$ in sucrose $5 \%$. Keep the dilution in the dark.

5. Dilute the bacterial solution (Table 1) at a 1:1 ratio with the $100 \mu \mathrm{M}$ R19-S probe solution. This mixture will constitute the inoculation solution.

a. The negative control batch is prepared by combining $25 \mu \mathrm{l}$ of the $100 \mu \mathrm{M}$ R19-S solution and $25 \mu \mathrm{l}$ of $5 \%$ sucrose.

b. The Btk batch is prepared by combining $25 \mu \mathrm{l}$ of $100 \mu \mathrm{M}$ R19-S and $25 \mu \mathrm{l}$ of the Btk solution $\left(\mathrm{OD}_{400}\right)$. The concentration of bacteria used is $1 \times 10^{8} \mathrm{CFU} / 5 \mathrm{~cm}^{2} / \mathrm{fly}$.

Note: Preparation of the R19-S mixture must be done under dark conditions.

6. Place a $20 \mathrm{~mm}$ filter disk on the top of the medium inside the Drosophila narrow vials (Figures $1 \mathrm{~A}$ and $1 \mathrm{~B})$.

7. Deposit the inoculation solution on the filter disk (Figure 1C).

8. Transfer 10 starved flies into one Drosophila narrow vial and allow the Drosophila to feed for 30 min on the inoculation solution (Figure 1D).

Note: For the 30 min time point, skip Step C9 below and dissect flies as described in Procedure $D$ below.

9. Remove flies from vials and put them into fresh vials with filter disks only soaked with $25 \mu \mathrm{l}$ of $100 \mu \mathrm{M}$ R19-S solution $+25 \mu \mathrm{l}$ of $5 \%$ sucrose. Let the flies feed until the time of dissection $(1 \mathrm{~h}$ and $1.5 \mathrm{~h})$.
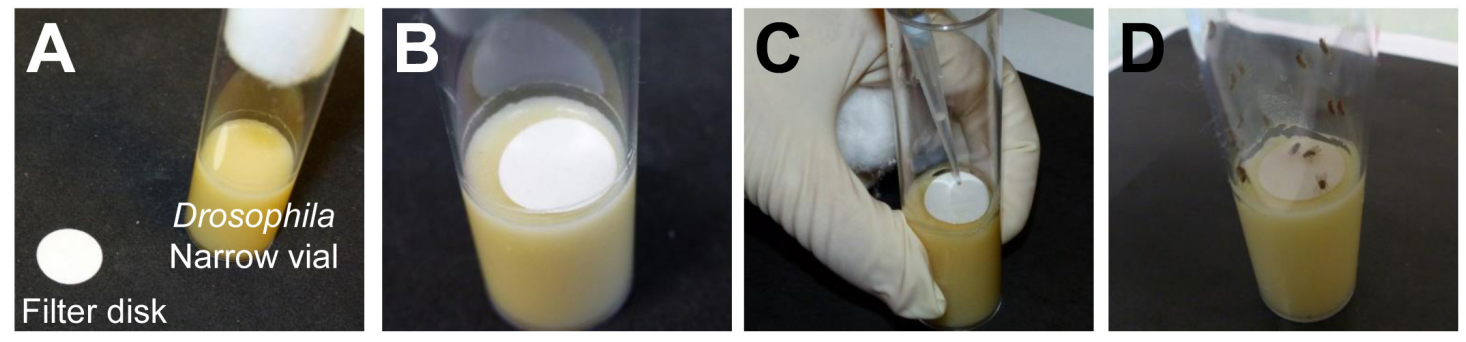

Figure 1. Inoculation procedure. A. Cut filter disks with a diameter of $20 \mathrm{~mm}$. B. Place on the top of the medium within the vial. C. Deposit the inoculation solution on the filter disk. D. Introduce the flies. 


\section{Dissection (Video 1)}

At the desired time points: $0.5 \mathrm{~h}, 1 \mathrm{~h}$ and $1.5 \mathrm{~h}$ start dissection.

1. Before starting, the forceps and dissecting watch glasses have to be rinsed with a $70 \%$ ethanol solution.

2. Ten females per condition are anesthetized with $\mathrm{CO}_{2}$ using the Drosophila-Sleeper.

3. Place one fly in a watch glass pre-filled with $1 \mathrm{ml} 1 x$ PBS (see Recipes).

4. Using forceps, pull the head away. Hold the fly gently and make an incision in the abdomen at the thorax/abdomen boundary. Gently separate the abdomen from the thorax. You should see the intestine still attached to both the thorax and the abdomen.

5. Then, the intestine is carefully stretched.

6. Cut the gut at the boundaries between foregut and hindgut. Remove the Malpighian tubules and/or the ovaries if still attached to the midgut at the midgut/hindgut boundary.

Note: If possible, dissection must be carried out in less than 30 s/intestine to avoid fading of the R19-S fluorescence.

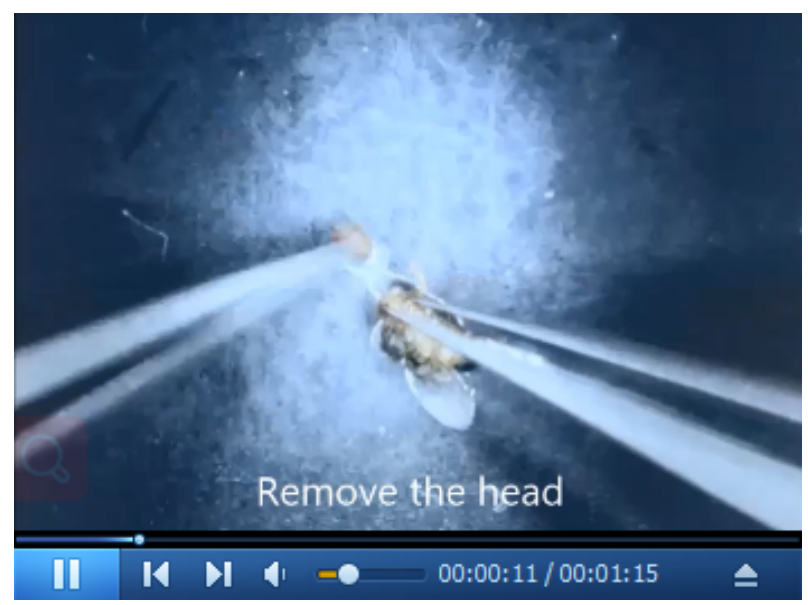

Video 1. Dissection procedure

E. Sample preparation and image capture

1. Fix Drosophila guts in $500 \mu \mathrm{l}$ of $4 \%$ formaldehyde in PBS (see Recipes) in 12-well plates at room temperature without agitation for 50 min in darkness.

Note: Cover the plate with aluminum foil to keep intestine samples in the dark.

2. Rinse twice with PBS $(2 \times 5 \mathrm{~min})$.

3. For each experiment, mount guts in Vectashield/DAPI and immediately observe the samples using a fluorescence microscope (Zeiss Axioplan Z1 with Apotome 2 microscope). R19-S fluorescence is visible within the "Rhodamine" emission range (excitation at $515 \mathrm{~nm}$ ). A differential interference contrast (DIC) image is also taken to merge with the fluorescence image. 
4. Take the pictures in the anterior part of midguts because $\mathrm{HOCl}$ is produced there. Below, in Figure 2, we present results for two conditions $\left(\mathrm{H}_{2} \mathrm{O}\right.$ and $\left.B t k\right)$. For each condition, we performed 3 (at $30 \mathrm{~min}$ and $1 \mathrm{~h}$ ) or 4 (at $1.5 \mathrm{~h}$ ) independent experiments.
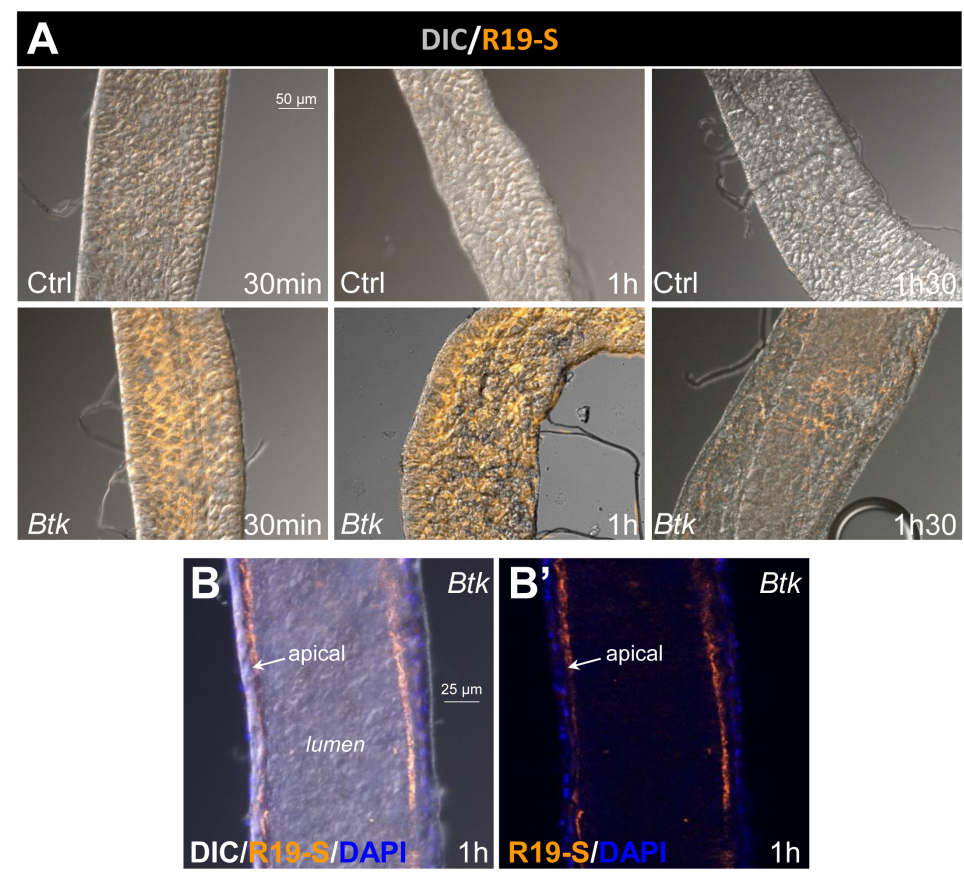

Figure 2. Image capture. A. Apotome image capture with the Rhodamine filter (orange) to visualize R19-S fluorescence and with the differential interference contrast (DIC) channel to outline intestine contours. Images were captured in the anterior part of the midgut. Ctrl: $5 \%$ sucrose negative control batch. Btk: Btk batch. B-B'. Cross section of anterior midgut at the level of the lumen. R19-S fluorescence (orange) is localized at the apical surface of the epithelium facing the lumen. Blue (DAPI) marks the epithelial nuclei. B incorporates the DIC image for orientation, whereas B' does not.

\section{Data analysis}

1. For each condition, analyze at least 3 independent experiments that have been performed on different days.

2. At least 20 intestines per condition have to be analyzable when you combine the three independent experiments. If this is not the case, you must carry out a supplementary experiment to reach the threshold of 20 intestines.

3. The percentage of R19-S-positive intestines is calculated for each experiment independently. An intestine is considered positive for R19-S labeling when at least $10 \%$ of the anterior midgut displays a fluorescent labeling outlining the enterocyte membranes. Scattered cells are not taken into account. 
Note: For each experiment, the number of R19-S-positive intestines under control conditions (fed with $5 \%$ sucrose) must not exceed $25 \%$ of the intestines. This would indicate that the flies underwent an unexpected stress before or during the experiments. Therefore, if more than $25 \%$ of the intestines are R19-S positive in the control experiment, discard all the experiments (control and bacteria-infected batch).

4. Pool the 3 (or more) independent experiments for each condition and compare as presented in the Excel figure below (Figure 3).

5. Statistical analysis

If $\mathrm{n}<30$ intestines, perform a non-parametric Kruskal and Wallis test using Kplot software.

If $\mathrm{n} \geq 30$ intestines, perform a parametric $t$-test using Excel or Kplot software.

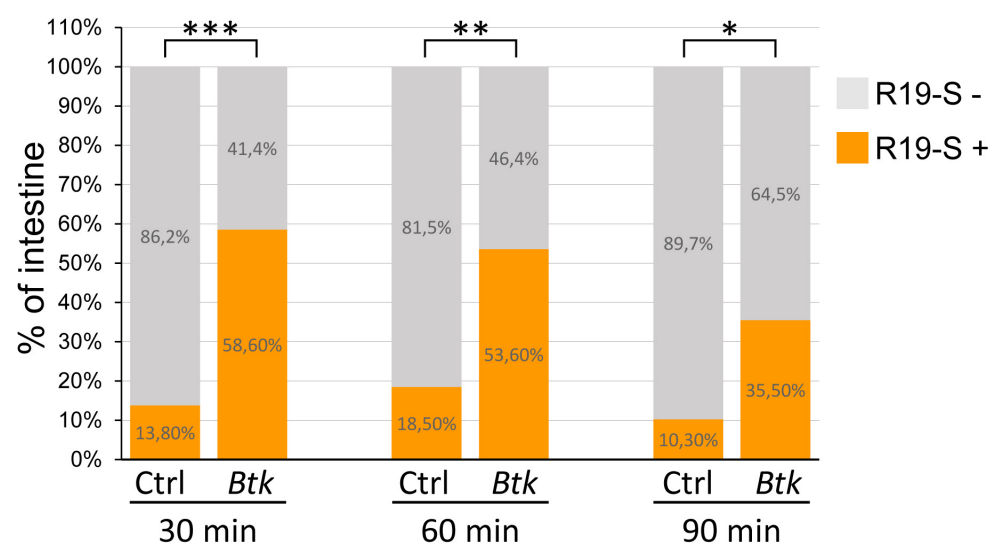

Figure 3. Percentage of R19-S positive midgut. R19-S-positive midguts are represented in orange and R19-S-negative midgut in grey. Data were acquired 30, 60 and 90 min postinoculation. Ctrl: Negative control batch. Btk: Btk batch. Since $27<n<31$, we used a nonparametric Kruskal and Wallis test.

\section{Recipes}

1. Standard nutrient medium for Drosophila melanogaster

Note: All the reagents are prepared with distilled water.

$8 \mathrm{~g} / \mathrm{L}$ agar

$25 \mathrm{~g} / \mathrm{L}$ sugar

$80 \mathrm{~g} / \mathrm{L}$ cornflour

$20 \mathrm{~g} / \mathrm{L}$ yeast

$6 \mathrm{~g} / \mathrm{L}$ tegosept (stock solution at $100 \mathrm{~g} / \mathrm{L}$ in $95 \%$ ethanol. Store at $4{ }^{\circ} \mathrm{C}$ )

2. LB medium
a. Weigh out $25 \mathrm{~g}$ of Luria broth medium powder
b. Adjust to $1 \mathrm{~L}$ with distilled water in a graduated cylinder
c. Adjust the $\mathrm{pH}$ to 7.2 if necessary
d. Autoclave 
3. LB-agar medium
a. Weigh out $25 \mathrm{~g}$ of Luria broth medium powder
b. Adjust to $1 \mathrm{~L}$ with distilled water in a graduated cylinder
c. Adjust the $\mathrm{pH}$ to 7.2 if necessary
d. Add $15 \mathrm{~g}$ agar powder
e. Autoclave

4. $5 \%$ sucrose

a. Weigh out $2.5 \mathrm{~g}$ of sucrose in a $50 \mathrm{ml}$ tube

b. Add $50 \mathrm{ml}$ sterile distilled water and vortex

Note: Make the solution just before use.

5. $1 \times$ phosphate-buffered saline (PBS)
a. Add $100 \mathrm{ml}$ of $10 x$ PBS solution to $900 \mathrm{ml}$ of distilled water in a graduated cylinder
b. Autoclave

6. $4 \%$ formaldehyde

Add $750 \mu$ of $1 \times$ PBS solution to $250 \mu$ l of $16 \%$ formaldehyde

7. $1 \mathrm{mM}$ R19-S

a. Prepare the stock solution at $1 \mathrm{mM}$ from the commercial powder in $100 \%$ acetonitrile

b. Store at $4{ }^{\circ} \mathrm{C}$ in the dark. Make aliquots if necessary

\section{Acknowledgments}

$\mathrm{SH}$ has been supported by the French government, through the UCA JEDI Investments in the Future project managed by the National Research Agency (ANR; reference number ANR-15-IDEX-01). OB was supported by INRA and AG by the CNRS. The procedure presented here was adapted from that of Lee and colleagues from the Won-Jae Lee Lab.

\section{Competing interests}

The authors have no conflicts of interest or competing interests.

\section{$\underline{\text { References }}$}

1. Benguettat, O., Jneid, R., Soltys, J., Loudhaief, R., Brun-Barale, A., Osman, D. and Gallet, A. (2018). The DH31/CGRP enteroendocrine peptide triggers intestinal contractions favoring the elimination of opportunistic bacteria. PLoS Pathog 14(9): e1007279.

2. Bonfini, A., Liu, X. and Buchon, N. (2016). From pathogens to microbiota: How Drosophila intestinal stem cells react to gut microbes. S0145-0305X(0116)30032-30035. 
3. Gonzalez, J. M., Jr., Brown, B. J. and Carlton, B. C. (1982). Transfer of Bacillus thuringiensis plasmids coding for delta-endotoxin among strains of $B$. thuringiensis and B. cereus. Proc Natl Acad Sci U S A 79(22): 6951-6955.

4. Kim, S. H. and Lee, W. J. (2014). Role of DUOX in qut inflammation: lessons from Drosophila model of gut-microbiota interactions. Front Infect Microbiol 3: 116.

5. Lee, K. A., Kim, S. H., Kim, E. K., Ha, E. M., You, H., Kim, B., Kim, M. J., Kwon, Y., Ryu, J. H. and Lee, W. J. (2013). Bacterial-derived uracil as a modulator of mucosal immunity and gutmicrobe homeostasis in Drosophila. Cell 153(4): 797-811.

6. Xia, X., Cheng, L., Zhang, S., Wang, L. and Hu, J. (2017). The role of natural antimicrobial peptides during infection and chronic inflammation. Antonie Van Leeuwenhoek 30(10): 0170929. 